La faune comprend 779 ossements animaux dont $71 \%$ ont pu être identifiés; ce pourcentage montre la très bonne conservation des éléments.

Nous avons tenté à travers cet échantillon d'aborder deux aspects liés aux ossements animaux : il s'agit pour le premier d'une quantification du dépôt s'appuyant sur les dénombrements des espèces animales et des pièces anatomiques, et pour le second d'une réflexion archéologique sur la qualification du type de dépôt. Ces deux aspects étudiés conjointement nous permettent d'aborder la faune de l'hôpital Sainte-Croix avec une problématique comparative, dont l'élément majeur sera la faune du site du Verbe-Incarné.

\section{LES DÉNOMBREMENTS}

Le porc domine très nettement tant en Nombre Minimum d'Individus (NMI), qu'en Nombre de Restes par Espèces (NRE) (avec respectivement $39 \%$ et $34 \%$ ) (tabl. I). Viennent ensuite les ovicaprinés et les bovinés dans des proportions équivalentes en NMI ; le bœuf est cependant mieux représenté en Nombre de Restes (NR) (qui approche celui du porc). Cette distorsion entre NMI et NRE de bœuf peut être expliquée par l'excessive fragmentation des os de cette espèce d'une part et par l'abondance relative de certaines pièces du squelette de bœuf d'autre part (fragments de crâne, vertèbres, par exemple), déficientes chez les autres. La distorsion est donc due à un problème méthodologique bien connu des archéozoologues (Poplin, 1976). Le chien, avec $11 \%$ en NMI est bien représenté, avec toutefois peu de restes $(2,7 \%)$. Le cheval, le cerf et le lièvre, avec respectivement $5 \%$ en NMI, peuvent être qualifiés ici d'espèces minoritaires au sein de l'échantillon.
Tabl. I - Nombre Minimum d'Individus (NM I) et Nombre de Restes par Espèces (NRE).

\begin{tabular}{|c|c|c|c|c|}
\hline ESPĖCE & NMI & $\%$ & NRE & $\%$ \\
\hline PORC $\ldots \ldots \ldots \ldots \ldots \ldots$ & 7 & 39 & 192 & 34 \\
\hline BEUUF . . . . . . . . . & 3 & 16 & 181 & 32 \\
\hline MOUTON/CHËVRE ......... & 3 & 16 & 109 & 19 \\
\hline CHIEN $\ldots \ldots \ldots \ldots \ldots \ldots \ldots$ & 2 & 11 & 15 & 2,7 \\
\hline CHEVAL ..... & 1 & 5 & 2 & 0,3 \\
\hline CERF ... & 1 & 5 & 1 & 0,1 \\
\hline LIÈVRE ........... & 1 & 5 & 2 & 0,1 \\
\hline TOTAL & 18 & & 502 & \\
\hline
\end{tabular}

LE PORC

C'est dans l'échantillon, l'animal le mieux représenté. La grande majorité des pièces anatomiques porte les traces de la boucherie et de la consommation. Les membres antérieurs et postérieurs sont bien représentés, avec un bon équilibre entre les différentes pièces qui les composent. Seul le fémur est un peu déficient. Les bas de pattes sont représentés uniquement par des métapodes, les phalanges étant inexistantes. La colonne vertébrale et les côtes sont très mal représentées, tout comme la tête.

Ce sont surtout les pièces les plus riches en viande qui ont été rejetées dans le fossé, après consommation. 


\section{Les âges}

Les âges ont été estimés à partir du degré d'épiphysation des os (Barone, 1976) $(\mathrm{n}=38)$.

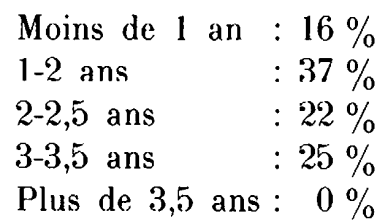

Une majorité d'animaux ont été abattus entre 1 an et 2 ans ( $37 \%)$, ce qui correspond aux individus parvenus à l'âge adulte. Cependant, les pourcentages sont assez élevés pour les classes d'âges déterminées ci-dessus; ceci contribue à une répartition assez régulière de l'abattage, partagée entre des individus tout juste adultes et des animaux adultes mais plus âgés.

Le sexe

La détermination du sexe des porcs a été effectuée sur des canines constituant toutefois un échantillon assez faible $(n=8)$, qui nous contraint à rester prudente quant à l'interprétation.

\begin{tabular}{|l|c|c|}
\hline & Femelles & Mâles \\
\hline Nombre des canines inférieures ....... & 2 & 5 \\
Nombre des canines supérieures ...... & 1 & 0 \\
NMI ........................ & 2 & 4 \\
\hline
\end{tabular}

Le NMI calculé à partir des canines $(\mathrm{NMI}=6)$ est assez proche de celui estimé sur les os $(\mathrm{NMI}=7)$ et donnerait une proportion de mâles deux fois plus importante que les femelles. Ceci refléterait une situation habituelle sur les sites de cette période.

\section{LES OVICAPRINÉS}

Le mouton et la chèvre n'ont pas pu être distingués. Les éléments qui permettent de les différencier ne nous ont pas semblé suffisants pour présenter des dénombrements : un seul radius a pu êlre altribué au moulon el là majorité des fragments de crâne semble lui appartenir. Ceci ne doit pas exclure a priori la présence de la chèvre.

Sur le plan de la répartition des pièces anatomiques, ce sont les os des membres qui sont les mieux représentés. La colonne vertébrale, les côtes et la tête sont très déficientes. Comme pour le porc, ce sont surtout les os longs qui ont été rejetés dans le fossé.

\section{Les âges}

Les âges ont été estimés à partir du degré d'épiphysation des os $(n=19)$. Deux classes ont été constituées, limitées par la faiblesse de l'échantillon. Elles nous permettent d'étudier une répartition dont l'image ne peut être que très générale.

Moins de 3-5 mois : $33 \%$

Moins de $25-35$ mois : $67 \%$

Une majorité d'animaux ont été abattus vers 2-3 ans et un nombre assez important d'individus avant de parvenir à l'âge adulte. Cette situation est très semblable à celle des ovicaprinés de Feurs par exemple et cadre bien avec les courbes observées sur la majorité des sites de l'époque.

\section{L.E BEUF}

Les os de bœuf sont assez nombreux mais très fragmentés. L'ensemble du squelette est bien représenté; on remarque un équilibre relatif entre toutes les pièces anatomiques, y compris la colonne vertébrale, les côtes et la tête. Ce sont les os des membres qui seraient relativement moins bien représentés, ce qui s'oppose à ce qui a été observé pour les autres espèces.

\section{Les âges}

Les âges ont été estimés à partir des épiphysations des os longs $(\mathrm{n}=26)$.

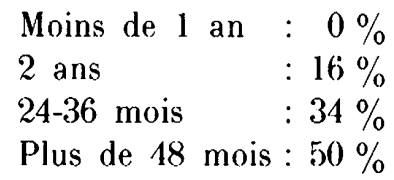

Une majorité d'animaux ont été abattus après 4 ans. Le reste se répartit entre des animaux abattus entre 2 ans et 2 ans et demi et quelques animaux abattus vers 2 ans. Aucun veau n'a été identifié.

Nous savons à partir de ces résultats que $50 \%$ des animaux sont abattus après 4 ans, sans précision possible. A cette époque, l'abattage moyen se situe après 6 ans (Feurs : 6 ans et demi), pour des animaux qui de toute évidence ont fourni produits (lait, reproduction) et force de travail (traction). Il est envisageable que les jeunes adultes abattus à I'hôpital Sainte-Croix aient été destinés précisément à la boucherie. On aurait donc un abattage qui se serait réparti entre des animaux destinés à la boucherie dès l'âge adulte et des animaux plus âgés abattus après réforme.

La détermination du sexe chez les bovinés, qui nous aurait permis d'affiner cette hypothèse, n'a pas pu être effectuée, faute d'échantillon statistique suffisant.

\section{LE CHIEN}

Il n'est représenté que par 15 pièces anatomi- 
ques, émanant pour la plupart. des os longs, à l'exception d'un axis et de quatre métapodes. C'est surtout le membre antérieur qui est représenté (11 pièces anatomiques sur les 15).

Nous avons pu relever sur deux humérus de nombreuses traces de découpe qui semblent correspondre à un écorchement (enlèvement de la peau). D'autres, observées sur les extrémités de l'ulna et du radius, pourraient être liées à la désarticulation des membres.

Le prélèvement de la peau des chiens est une activité courante sur les sites de La Tène finale (Yvinec, 1987) : Villeneuve-Saint-Germain (Aisne), Levroux (Indre), Feurs (Loire) ... II n'est pas possible de préciser ici si les traces d'écorchement relevées sur les deux humérus résultent de l'enlèvement de la peau en vue de la préparation des carcasses pour la consommation, ou si seule la fourrure était recherchée.

Il n'en demeure pas moins que les carcasses des chiens ont subi un traitement, sur lequel nous n'avons que très peu d'informations. La consommation de cet animal ne doit pas être écartée; elle a été mise en évidence sur la plupart des sites gaulois.

\section{LE CHEVAL}

Il n'est représenté que par une dent et un fragment de phalange proximale. Sauf cas exceptionnel (sur les sites cultuels par exemple), le cheval est l'animal domestique le moins bien représenté sur les sites d'habitat. Ces animaux sont donc rarement consommés, comme semble l'indiquer leur quasiabsence dans les dépotoirs de consommation.

\section{LE CERF}

Cette espèce est représentée par un unique fragment de maxillaire. Sa présence nous indique toutefois une activité de chasse.

\section{LE LIÈVRE}

Il n'est représenté que par deux pièces anatomiques pouvant appartenir au même individu et, comme pour le cerf, on ne peut que rapporter sa présence à la chasse.

\section{LA BIOMÉTRIE ${ }^{1}$}

Le matériel étant fragmenté et trop peu nombreux, l'étude de la morphologie des espèces est

1 Les données biométriques brutes non publiées ici sont disponibles auprès de l'auteur, sur demande. limitée. Quelques tailles au garrot, estimées pour le porc et le chien uniquement, peuvent nous permettre de comparer grossièrement la morphologie de ces espèces par rapport à celles des sites contemporains.

Le porc (tabl. II)

Trois hauteurs ont été estimées (coefficients de Teichert, 1875) à partir de trois métapodes :

Métacarpe III $=71,8 \mathrm{~cm}$
Métacarpe $I I I=77,1 \mathrm{~cm}$
Métatarse IV $=76 \quad \mathrm{~cm}$

A partir de ces trois données (à considérer avec précaution car peu nombreuses), on note une homogénéité relative. La moyenne de ces données est de $74,9 \mathrm{~cm}$. Ces animaux semblent assez proches de ceux du Verbe-Incarné et de ceux de Feurs. Ils sont également très semblables aux porcs européens dont les tailles sont homogènes en général.

Tabl. II - Données de comparaisons sur la taille au garrot $(\mathrm{en} \mathrm{cm})$ des porcs à l'Age du Fer.

\begin{tabular}{|c|c|c|c|}
\hline Site & Moyenne & Étendue & Référence \\
\hline Beauvais (Oise) & 69,7 & $60,7-87,1$ & Méniel, 1984 \\
\hline Variscourt (Aisne) . . . . . & 70,1 & $55,1-79$ & Méniel, 1984 \\
\hline Levroux (Indre) ........ & 73,8 & $60-81$ & Krausz, 1985 \\
\hline Manching (Allemagne) .. & 69,6 & $58-80$ & $\begin{array}{l}\text { Bœssneck } \\
\text { et al., } 1971\end{array}$ \\
\hline Feurs (Loire) ........ & 66,6 & $60,2-71,9$ & Vila, 1988 \\
\hline $\begin{array}{l}\text { Lyon, Verbe-Incarné } \\
\text { (Rhône) } \ldots \ldots \ldots \ldots \ldots\end{array}$ & 72,5 & $68-80$ & Krausz, 1989 \\
\hline
\end{tabular}

Le chien (tabl. III)

Deux hauteurs ont été estimées à partir de deux radius (coefficient de Koudelka, 1885) et donnent : 47 et $57,9 \mathrm{~cm}$.

Nous avons donc deux individus de bonne taille, avec une variation de $10 \mathrm{~cm}$.

Les tailles des chiens à la fin de La Tène sont assez hétérogènes et se rapportent au phénomène à présent bien connu de la multiplication des races qui commence à cette époque. Il existait des individus très petits (Variscourt : $22 \mathrm{~cm}$ ), des animaux très grands (Levroux : $57,5 \mathrm{~cm}$ ) et une infinité de tailles intermédiaires.

Tabl. III - Données de comparaisons sur les tailles au garrot (en $\mathrm{cm})$ des chiens à la fin de l'Age du Fer.

\begin{tabular}{|c|c|c|}
\hline Site & Étendue & Référence \\
\hline Variscourt (Aisne). & $22-52$ & Méniel, 1984 \\
\hline Beauvais (Oise) . . & $38-52$ & Méniel, 1984 \\
\hline Manching (Allemagne) ..... & $32,5-52$ & $\begin{array}{l}\text { Boessneck et al., } \\
1971\end{array}$ \\
\hline Levroux (Indre). . & $40-57$ & Krausz, 1985 \\
\hline
\end{tabular}




\section{Les autres espèces}

Pour les autres espèces, aucune étude biométrique n'est envisageable. Nous insisterons toutefois brièvement sur le cas du bœuf : pour cette période basse de La Tène qui caractérise le site de l'hôpital Sainte-Croix, nous nous sommes interrogée sur la présence du "grand bœuf». Des mesures prises sur quelques phalanges proximales ont été comparées à celles des bœufs de Feurs, qui ont été identifiés par E. Vila comme représentant bien les petits bœufs indigènes. Les données de l'hôpital Sainte-Croix sont parfaitement homogènes avec celles de Feurs. Le "grand bœuf d'importation" est donc absent de notre échantillon (tabl. IV).

Tabl. IV - Diagramme de dispersion des bocufs d'après la biométrie de la phalange 2 : mesure de la Longueur totale (L.t) et du Diamètre Transverse Proximal (DTP). Sites de Feurs et de l'hôpital Sainte-Croix.

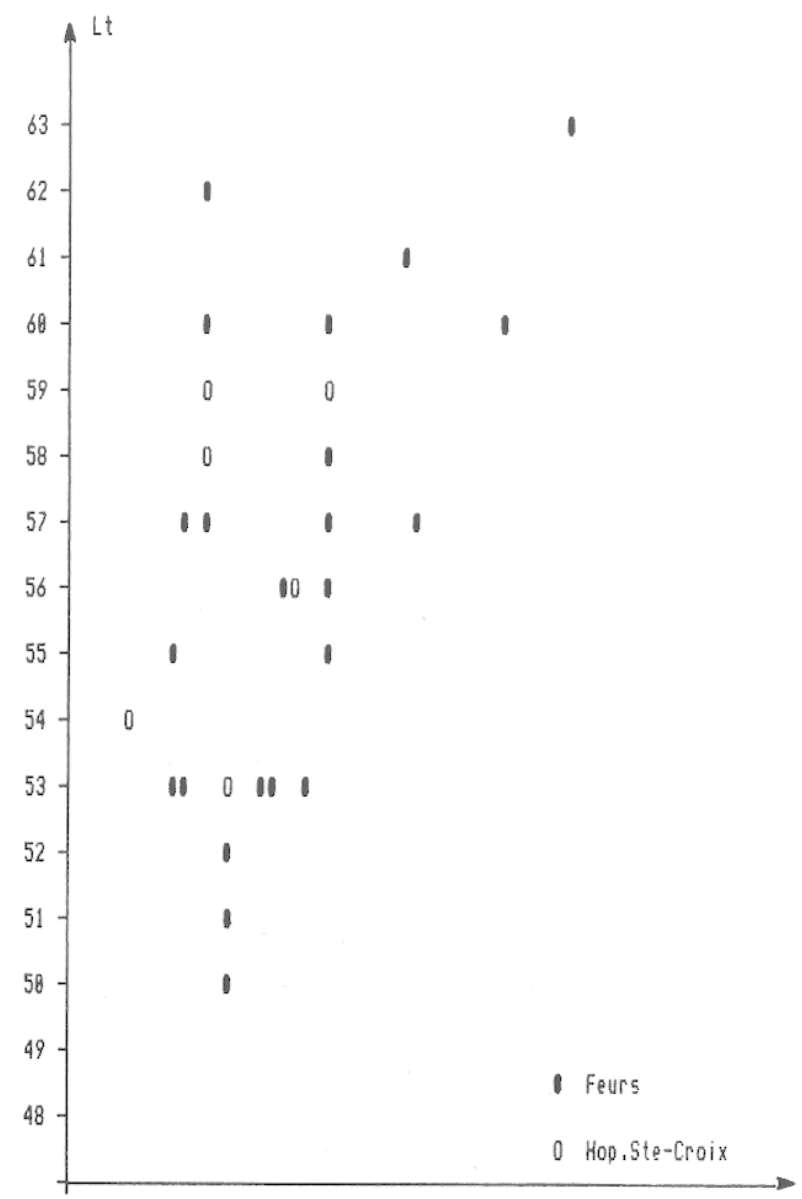

$\begin{array}{llllllllllllll}21 & 22 & 23 & 24 & 25 & 26 & 27 & 28 & 29 & 38 & 31 & 32 & 33 & \text { OTP }\end{array}$

\section{LES ÉCLATS DE DÉBITAGE}

Nous avons regroupé ici des restes non identifiables, mais présentant les caractéristiques d'un débitage. Ces éléments représentent environ $13 \%$ du Nombre Total de Restes $(n=108)$.

Les éclats ont été divisés en deux groupes :

- ceux qui appartiennent au petit bétail (ovicaprinés et porc), soit $6 \%$ du Nombre Total de Restes $(n=50)$,

- ceux qui appartiennent au grand bétail (bœuf principalement, éventuellement au cerf, le cheval s'excluant de lui-même), soit $7 \%$ du Nombre Total de Restes $(n=58)$.

Ces éclats semblent caractériser surtout un débitage de boucherie (l'artisanat ne devant toutefois pas être écarté). Ce matériel nous permet de mettre en évidence la présence de déchets de boucherie à distinguer des résidus de cuisine.

\section{LA DÉCOUPE}

Un relevé systématique a été effectué sur chaque espèce, conformément à l'étude du système de découpe des porcs du Verbe-Incarné. Les données obtenues sur la faune de l'hôpital Sainte-Croix sont trop ténues pour permettre de reconstituer des systèmes de découpe, comme nous avions pu le faire pour les porcs du Verbe-Incarné (Krausz, 1989). Une description de toutes nos observations nous éloignerait $\mathrm{du}$ but de cet article et nous préférerons, en guise de comparaison, souligner quelques points de la découpe des porcs du Verbe-Incarné et de celle qui semble s'esquisser à l'hôpital Sainte-Croix.

La colonne vertébrale

Hôpital Sainte-Croix : il existe plusieurs cas de vertèbres (lombaires) tranchées par le milieu, dans le plan sagittal.

Verbe-Incarné : cette technique de découpe n'a pas été observée.

\section{Le membre antérieur}

Hôpital Sainte-Croix : l'humérus est coupé transversalement par le milieu, ainsi que l'ulna et le radius. La désarticulation au couteau de l'ulna de l'humérus est bien visible.

Verbe-Incarné : l'humérus est coupé le plus souvent par le milieu; l'ulna et le radius sont en général intacts, la viande ayant été désossée.

\section{Le membre postérieur}

Hôpital Sainte-Croix : le bassin est fracturé en trois parties dégageant l'illium, l'ischium et l'acetabulum. Le fémur cst fracturé à tous les niveaux de la 
diaphyse sans que les épiphyses ne soient jamais touchées. Le tibia est fracturé à tous les niveaux de la diaphyse également, ou parfois longitudinalement dans l'axe de la crête tibiale. Les épiphyses ne sont jamais touchées.

Verbe-Incarné : même découpe qu'à l'hôpital Sainte-Croix pour le bassin. Le fémur est souvent découpé transversalement au milieu de la diaphyse. Le tibia est, quant à lui, dans la plupart des cas complet el vierge de traces.

La technique de découpe des porcs du VerbeIncarné est très systématisée, précise et comporte nombre de caractères particuliers qui n'ont pas été observés à l'hôpital Sainte-Croix. Sur ce dernier site, la découpe des porcs (ainsi que celle des autres espèces) semble assez anarchique, comme nous le constatons en général sur les sites d'habitat de La Tène finale.

\section{Comparaisons (tabl. V)}

\section{Verbe-Incarné}

Sur le plan de la faune, le fossé du VerbeIncarné s'oppose à celui de l'hôpital Sainte-Croix : nous disposions en effet au Verbe-Incarné d'un dépôt de porcs émanant d'un rejet global comprenant, en parts équivalentes, des détritus provenant des différentes opérations de découpage liées à la boucherie et à la cuisine. Nous avions démontré que les animaux avaient été abattus, découpés et consommés sur place; l'ensemble des déchets se trouvait au même endroit.
Il n'en est rien à l'hôpital Sainte-Croix : nous n'avons pas de trace évidente de l'abattage et le rapport entre la part des résidus de boucherie et ceux de la cuisine n'est pas équilibré.

La faune du Verbe-Incarné est constituée essentiellement par un troupeau de porcs $(85 \%$ en $\mathrm{NMI})$, qui ne laisse pas de place aux autres espèces habituelles. Elle relève donc d'une gestion de troupeau particulière, composée d'une espèce unique, le porc, qui ne peut pas refléter un troupeau d'habitat. La faune de l'hôpital Sainte-Croix se compose d'espèces variées, présentes dans des proportions habituelles aux sites de La Tène finale. La faune du Verbe-Incarné concerne de toute évidence un ensemble particulier qui ne trouve actuellement aucun parallèle en Europe.

\section{Feurs}

Sur les cinq fosses récemment publiées du site de Feurs, la fosse 5 présente précisément la même composition de faune qu'à l'hôpital Sainte-Croix (pour un nombre de restes quasi équivalent). Les animaux occupent les mêmes places au sein du troupeau, avec les mêmes espèces minoritaires (lièvre et cheval, le cerf n'étant pas représenté). Avec Feurs, les parallèles sont nombreux : les âges d'abattage pour les trois espèces principales suivent le même schéma et les quelques hauteurs au garrot calculées concordent bien elles aussi. Il semble également que le bouf de Feurs soit très proche de celui de l'hôpital Sainte-Croix.

Tabl. V - Comparaisons des Nombres Minimum d'Individus en pourcentages.

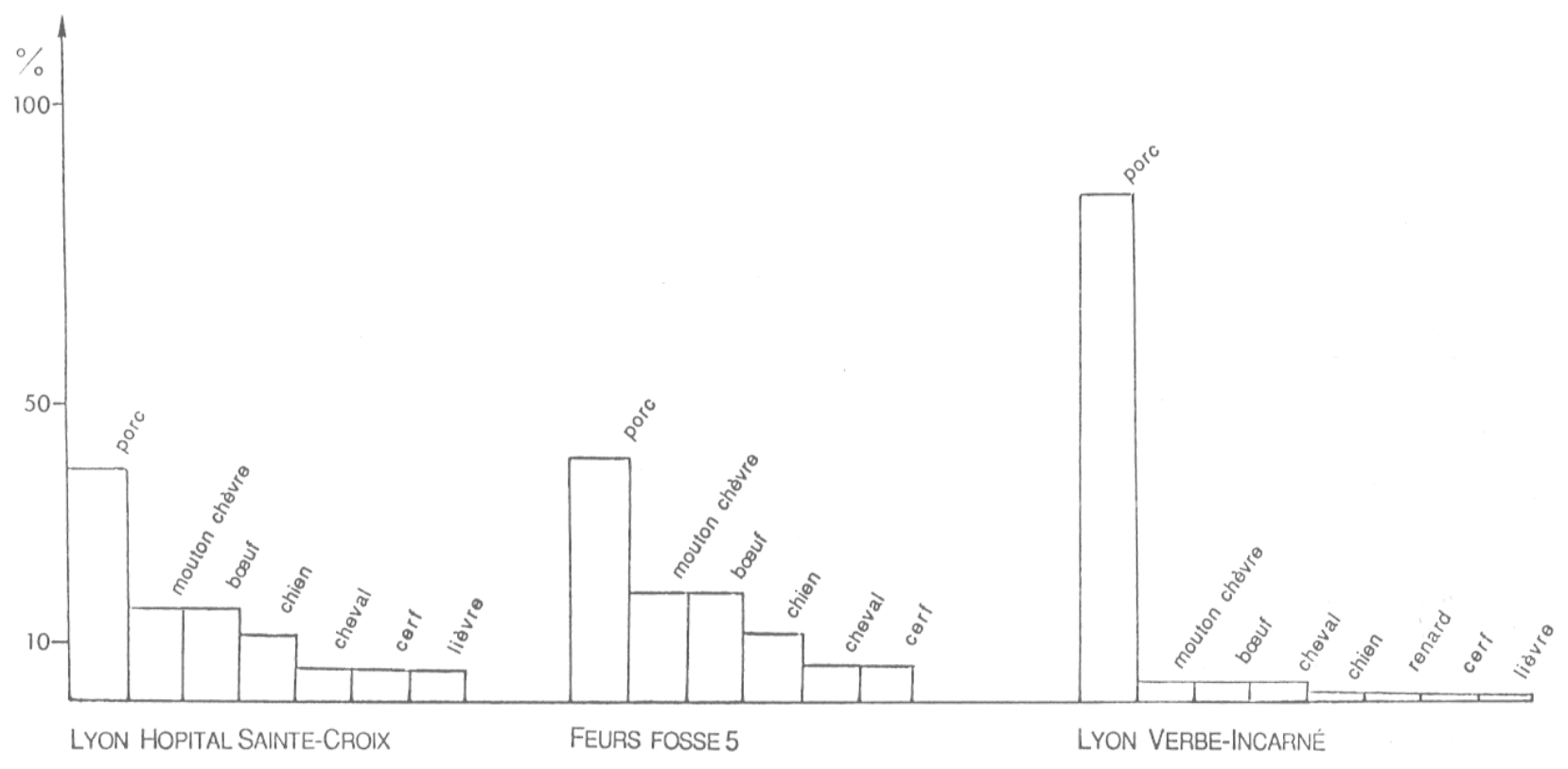




\section{Autres sites français}

Si l'on considère les faunes qui proviennent des grands sites d'habitat de La Tène finale (Levroux, Variscourt, Villeneuve-Saint-Germain par exemple). Il apparaît sur l'ensemble une relative homogénéité des troupeaux, avec toujours le porc comme espèce principale, suivi des ovicaprinés et des bovinés.

Le site de l'hôpital Sainte-Croix s'inscrit parfaitement dans la situation générale, ce qui nous conduit à penser, que d'après la faune, le type d'occupation du site est bien celui d'un habitat. Par comparaison également, la faune relève d'un élevage et d'une consommation bien typique de La Tène finale.

\section{Qualification DU TYPE DE DÉPôT}

Il ressort clairement de notre étude que ce sont les déchets des principales pièces de viande qui ont été rejetés dans le fossé après consommation, ceci pour toutes les espèces (nous n'y incluons pas les espèces minoritaires, cheval, cerf et lièvre qui font ici figure de faune résiduelle). Ce dépôt se compose surtout de déchets de cuisine. Toutefois, il convient d'y ajouter un ensemble de résidus de boucherie plus restreint que le rejet principal; ces résidus ont été mis en évidence par la découpe de certaines catégories anatomiques et par les éclats de débitage.

Par comparaison, la faune de l'hôpital SainteCroix trouve de nombreux parallèles avec les faunes d'habitat de la fin de l'Age du Fer du point de vue de sa composition. Elle n'a rien de commun avec celle du Verbe-Incarné, qui concerne un dépôt particulier, alors qu'elle reflète bien un troupeau et une consommation habituels de La Tène finale.

\section{Sophie Krausz*}

\footnotetext{
* A.D.E.L., 2, rue Traversière, 36100 Levroux.
}

\section{BIBLIOGRAPHIE}

\section{Barone R}

1976: Anatomie comparée des mammifères domestiques, I, Ostéologie, fasc. 2 Atlas, Paris, Éd. Vigot.

Boessneck J., von den Dsriesch A., Meyer-Lempennau U., Wechsler von Ohlen E.

1971: Die Tierknochenfunde aus dem Oppidum von Manching, Wiesbaden, F. Steiner.

\section{Krausz S.}

1985 : Les ossements animaux du Village des Arènes à Levroux, Indre. Le terrain Lacotte. Contribution à l'étude de la faune à La Tène finale, Mémoire de maîtrise, Univ. Paris I, 1985.

1989 : Aux origines de Lyon. Les fossés du plateau de la Sarra, les ossements animaux, Documents d'Archéologie en RhôneAlpes, 2, série lyonnaise 1, p. 44-50.
Méniel P.

1984 : Contribution à l'histoire de l'élevage en Picardie. Dou Néolithique à la fin de l'Age du Fer, Revue Archéologique de Picardie, no spécial.

\section{Poplin Fr.}

1976 : A propos du nombre de restes et du nombre d'individus dans les échantillons d'ossements, Cahiers du Centre de Recherches Préhistoriques de l'Université de Paris I, 5, p. 61-74.

\section{Vila E.}

1988: La faune in : Vaginay M., Guichard V., L'habital gaulois de Feurs, Documents d'Archéologie Française, 14, Paris, Éd. de la Maison des Sciences de l'Homme, p. 171-180.

Yvinec J.-H.

1987 : Découpe, pelleterie et consommation des chiens gaulois à Villerieuve-Saint-Germain, in: La découpe et le parlage du corps à travers le temps et l'espace, Anthropozoologica, $\mathrm{n}^{\text {" spécial, }}$ p. $83-90$. 